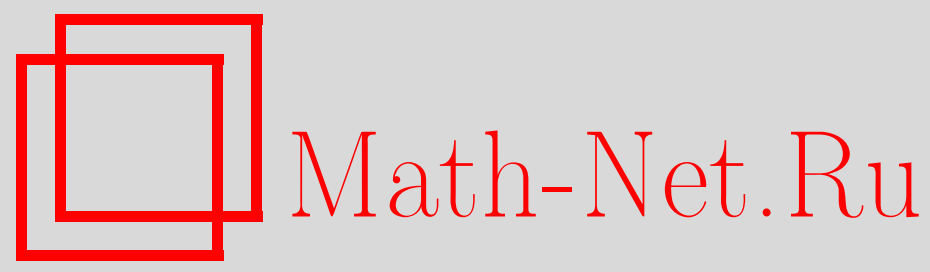

А. М. Гомилко, Г. В. Радзиевский, О числовых образах семейства коммутирующих операторов, Матем. заметки, 1997, том 62, выпуск 5, 787-791

DOI: https://doi.org/10.4213/mzm1665

Использование Общероссийского математического портала Math-Net.Ru подразумевает, что вы прочитали и согласны с пользовательским соглашением http://www.mathnet.ru/rus/agreement

Параметры загрузки:

IP : 18.234 .197 .8

26 апреля 2023 г., 14:45:55

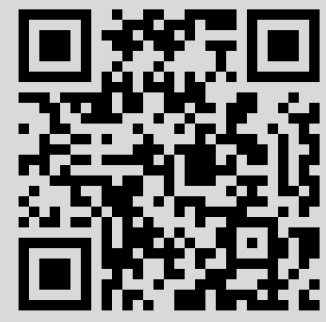




\section{О ЧИСЛОВЫХ ОБРАЗАХ СЕМЕЙСТВА КОММУТИРУЮЩИХ ОПЕРАТОРОВ}

\section{А. М. Гомилко, Г. В. Радзиевский}

Пусть $B$ - комплексное банахово пространство. Все операторы предполагаются линейньми, ограниченными и действующими из $B$ в $B$. Введем определение числового образа оператора [1]. По теореме Хана-Банаха для любого элемента $y \in B$ найдется такой линейньй непрерывный функционал (по крайней мере один) $f_{y} \in B^{*}$, что $f_{y}(y)=\|y\|^{2}$ и $\left\|f_{y}\right\|=\|y\|$. Выбор функционала $f_{y}$ для каждого $y \in B$ позволяет определить на $B$ полувнутреннее произведение $[\cdot, \cdot]$ по правилу $[x, y]=f_{y}(x), x, y \in B$. Тогда числовым образом оператора $A$ называется множество

$$
W(A):=\left\{[A x, x]: x \in B,[x, x]=\|x\|^{2}=1\right\} .
$$

Если $B$ - гильбертово пространство, то это определение совпадает с обычным определением числового образа. Однако (в отличие от случая гильбертова пространства), числовой образ оператора, действуюшего в банаховом пространстве, может быть невыпукльг [1] и, вообще говоря, зависит от выбора функционала $f_{y}$. Тем не менее, как показано в [2], выпуклая оболочка conv $\overline{W(A)}$ замыкания $\overline{W(A)}$ не зависит от $f_{y}$, определяющих полувнутреннее произведение на $B$.

Пусть $\sigma(A)$ - спектр оператора $A$, а $r(A)$ - его спектральньй радиус. Тогда $\sigma(A) \subseteq$ conv $\overline{W(A)}$, т.е. conv $\sigma(A) \subseteq \operatorname{conv} \overline{W(A)}$. Очевидно, что спектр оператора не зависит от замены исходной нормы банахова пространства $B$ на эквивалентную ей норму. Однако, такие характеристики оператора, как его норма и числовой образ, могут существенно измениться при замене исходной нормы на эквивалентную ей. Данная заметка посвящена изучению вопроса об изменении числовых образов семейств коммутирующих операторов при изменении исходной нормы. Далее символы $\|A\|_{*}$ и $W\left(A,\|\cdot\|_{*}\right)$ обозначают соответственно норму и числовой образ оператора $A$, вычисленные в норме $\|\cdot\|_{*}$. При этом, как было отмечено, включение $\sigma(A) \subseteq \operatorname{conv} \overline{W\left(A,\|\cdot\|_{*}\right)}$ устанавливает наименьшую из возможных границ изменения вьпуклой линейной оболочки замыкания числового образа оператора $A$ при эквивалентной перенормировке $\|\cdot\|_{*}$ банахова пространства. Простые примеры показывают, что равенство conv $\overline{W\left(A,\|\cdot\|_{*}\right)}=\operatorname{conv} \sigma(A)$ в общем случае не справедливо ни при какой эквивалентной перенормировке (в частности, для квазинильпотентного оператора). Поэтому следующая теорема в определенном смысле точна.

ТЕОрема 1. Пусть $A_{1}, \ldots, A_{n}$ - попарно коммутирующие операторы, действующие в банаховом пространстве $B$. Тогда для любого $\varepsilon>0$ найдется такая норма $\|\cdot\|_{\varepsilon}$, эквивалентная исходной, что числовой образ каждого из операторов $A_{1}, \ldots, A_{n}$ лежит в $\varepsilon$-окрестности выпуклой оболочки спектра этого оператора, m.e.

$$
W\left(A_{\gamma},\|\cdot\|_{\varepsilon}\right) \subset\left\{\lambda:|\lambda-\mu|<\varepsilon, \mu \in \operatorname{conv} \sigma\left(A_{\gamma}\right)\right\}, \quad \gamma=1, \ldots, n .
$$

Работа выполнена при частичной поддержке Фонда фундаментальных исследований при Государственном комитете Украины по вопросам науки и технологий. 
Если банахово пространство В является гильбертовым, то соответствующая норма $\|\cdot\|_{\varepsilon}$ может быть выбрана гильбертовой.

Далее $I$ - тождественный оператор в $B$ и $A^{0}:=I$. Доказательству теоремы 1 предпошлем лемму, распространяющую на случай $n$ операторов хорошо известньй факт, относящийся к изменению нормы оператора при эквивалентной перенормировке пространства.

Лемма. Пусть $A_{1}, \ldots, A_{n}$ - попарно коммутирующие операторы, действующие в банаховом пространстве В. Тогда для любого в>0 найдется такая норма $\|\cdot\|_{\varepsilon}$, әквивалентная исходной, что

$$
\left\|A_{\gamma}\right\|_{\varepsilon}<r\left(A_{\gamma}\right)+\varepsilon, \quad \gamma=1, \ldots, n
$$

Если банахово пространство В является гильбертовым, то соответствующая норма $\|\cdot\|_{\varepsilon}$ может быть выбрана гильбертовой.

ДокАЗАТЕЛЬСтво. Ряды $\sum_{k=0}^{\infty} R_{\gamma}^{-k}\left\|A_{\gamma}^{k}\right\|$ сходятся при $R_{\gamma}>r\left(A_{\gamma}\right)$, поэтому для $\bar{R}=\left(R_{1}, \ldots, R_{n}\right)$ норма $\|\cdot\|_{\bar{R}}$, заданная равенством

$$
\|x\|_{R}=\left(\sum_{k_{1}=0}^{\infty} \cdots \sum_{k_{n}=0}^{\infty} \frac{\left\|A_{1}^{k_{1}} \cdots A_{n}^{k_{n}} x\right\|^{2}}{R_{1}^{2 k_{1}} \cdots R_{n}^{2 k_{n}}}\right)^{1 / 2},
$$

определена для всех элементов $x \in B$, если только $R_{\gamma}>r\left(A_{\gamma}\right)$, причем норма $\|\cdot\| \|_{R}$ эквивалентна исходной норме $\|\cdot\|$. Очевидно также, что если исходная норма $\|\cdot\|$ была гильбертовой, то норма $\|\cdot\|_{\bar{R}}$ гильбертова. Из определения $\|\cdot\|_{\bar{R}}$ следуют неравенства $\left\|A_{\gamma} x\right\|_{\bar{R}} \leqslant R_{\gamma}\|x\|_{\bar{R}}, \gamma=1, \ldots, n$. Выбирая $R_{\gamma}=r\left(A_{\gamma}\right)+\varepsilon / 2$, получаем утверждение леммы.

Приведем следствие из леммы, относяшееся к мере обусловленности оператора $A$, т.е. к величине $\|A\| \cdot\left\|A^{-1}\right\|$, определенной в предположении ограниченной обратимости оператора $A$ (см., например, [3, с. 237]).

СлЕДСТВИЕ 1. Пусть $A$ - ограниченно обратимый оператор, действующий $в$ банаховом пространстве $B$. Тогда для любого $\varepsilon>0$ найдется такая норма, эквивалентная исходной, что мера обусловленности оператора $A$, вычисленная $в$ этой норме, меньше чем $r(A) r\left(A^{-1}\right)+\varepsilon$. Если банахово пространство $B$ является гильбертовым, то соответствующая норма моэсет быть выбрана гильбертовой.

ДОКАЗАТЕЛЬСТВО ТЕОРЕМЫ 1 . Пусть $\Psi_{r}(\alpha)=\{\lambda:|\lambda-\alpha| \leqslant r\}-$ круг с центром в точке $\alpha \in \mathbb{C}$ радиуса $r>0$. Из теоремы об аппроксимации выпуклых компактов [4, c. 18] следует, что для произвольного $\varepsilon>0$ найдутся такие круги $\Psi_{r_{\gamma, m}}(\alpha \gamma, m)$, для которых вьпуклая оболочка спектра каждого оператора $A_{\gamma}$ лежит внутри пересечения кругов $\Psi_{r_{\gamma, m}}\left(\alpha_{\gamma, m}\right), m=1, \ldots, s_{\gamma}$, а само это пересечение находится в $\varepsilon$-окрестности выпуклой оболочки спектра оператора $A_{\gamma}$, т.е.

$$
\operatorname{conv} \sigma\left(A_{\gamma}\right) \subset \bigcap_{m=1}^{s_{\gamma}} \Psi_{r_{\gamma, m}}\left(\alpha_{\gamma, m}\right) \subset\left\{\lambda:|\lambda-\mu|<\varepsilon, \mu \in \operatorname{conv} \sigma\left(A_{\gamma}\right)\right\}, \quad \gamma=1, \ldots, m \text {. }
$$


Согласно первому включению спектр каждого оператора $A_{\gamma}+\alpha_{\gamma, m} I$ лежит строго внутри круга $\Psi_{r_{\gamma, m}}(0)$ (с центром в нуле). Применяя теперь к коммутируюшим оператоpaм $A_{\gamma}+\alpha_{\gamma, m} I, \gamma=1, \ldots, n, m=1, \ldots, s_{\gamma}$, лемму, получаем существование такой нормы $\|\cdot\|_{\varepsilon}$, для которой $\left\|A_{\gamma}+\alpha_{\gamma, m} I\right\|_{\varepsilon}<r_{\gamma, m}$, а значит, если $\lambda \in W\left(A_{\gamma}+\alpha_{\gamma, m} I,\|\cdot\|_{\varepsilon}\right)$, то $|\lambda|<r_{\gamma, m}$. Поэтому числовой образ $W\left(A_{\gamma},\|\cdot\|_{\varepsilon}\right)$ оператора $A_{\gamma}$ находится в пересечении кругов $\Psi_{r_{\gamma, m}}\left(\alpha_{\gamma, m}\right), m=1, \ldots, s_{\gamma}$. Отсюда и из второго включения в соотношениях (1) получаем утверждение теоремы.

Приведенные далее два утверждения являются следствиями из теоремы 1 , которые распространяют на банахово пространство результаты, установленные ранее в случае гильбертова пространства.

СлЕДСтвИЕ 2 [5, теорема 4]. Пусть $A$ - ограниченный оператор, действующий в банаховом пространстве $B$. Тогда $\operatorname{conv} \sigma(A)=\bigcap$ conv $\overline{W\left(A,\|\cdot\|_{*}\right)}$, где пересечение берется по всем әквивалентным нормам $\|\cdot\|_{*}$ банахова пространства $B$. Если банахово пространство В является гильбертовым, то соответствующее пересечение берется по всем гильбертовым нормам $\|\cdot\|_{*}$.

СлЕДСТВИЕ 3 [6, с.95]. Для того чтобы спектры попарно коммутирующих операторов $A_{1}, \ldots, A_{n}$ лежсали внутри левой полуплоскости необходимо и достаточно существование такой нормы $\|\cdot\|_{*}$, әквивалентной исходной, что множества $\overline{W\left(A_{1},\|\cdot\|_{*}\right)}, \ldots, \overline{W\left(A_{n},\|\cdot\|_{*}\right)}$ лежсали бы внутри левой полуплоскости. Если банахово пространство $B$ является гильбертовым, то соответствующая норма $\|\cdot\|_{*}$ можсет быть выбрана гильбертовой.

ЗАмЕчАниЕ 1 . Пусть $A_{\gamma}$ - относительно компактное в операторной топологии множество попарно коммутирующих операторов. Тогда для произвольного $\varepsilon>0$ в нем найдется конечная $\varepsilon$-сеть $A_{\gamma_{1}}, \ldots, A_{\gamma_{n}}$. Применим к операторам $A_{\gamma_{1}}, \ldots, A_{\gamma_{n}}$ утверждение леммы и заметим, что указанная в этой лемме норма обладает свойством $\|D\|_{\varepsilon} \leqslant\|D\|$ для любого оператора $D$, коммутируюшего с $A_{\gamma_{1}}, \ldots, A_{\gamma_{n}}$. Отсюда получаем, что утверждение леммы справедливо и для бесконечного числа попарно коммутирующих операторов, образующих относительно компактное множество. Понятно также, как теорема 1 и следствие 3 распространяются на множества таких операторов.

Покажем существенность требования коммутируемости операторов $A_{\gamma}$ для справедливости теоремы 1 . Для этого рассмотрим в двумерном пространстве $\mathbb{C}^{2}$ операторы $A_{1}=\left(\begin{array}{ll}0 & 1 \\ 0 & 0\end{array}\right)$ и $A_{2}=\left(\begin{array}{ll}0 & 0 \\ 1 & 0\end{array}\right)$ с $\sigma\left(A_{1}\right)=\sigma\left(A_{2}\right)=\{0\}$. Если бы утверждение теоремы 1 для $A_{1}$ и $A_{2}$ было бы справедливьгм, то нашлась бы такая норма $\|\cdot\|_{*}$ в пространстве $\mathbb{C}^{2}$, что множества $W\left(A_{1},\|\cdot\|_{*}\right)$ и $W\left(A_{2},\|\cdot\|_{*}\right)$ лежали бы внутри круга $\{\lambda:|\lambda|<1 / 4\}$. Но тогда множество $W\left(A_{1}+A_{2},\|\cdot\|_{*}\right)$ принадлежало бы кругу $\{\lambda:|\lambda|<1 / 2\}$, что невозможно, так как $\sigma\left(A_{1}+A_{2}\right)=\{-1,1\}$. Этот же пример показьвает существенность требования коммутируемости операторов для справедливости леммы. Для следствия 3 такие примеры строятся полностью аналогично.

Установим критерий вьполнения включений $W\left(A_{\gamma},\|\cdot\|_{*}\right) \subseteq \Omega_{\gamma}$, где $\Omega_{\gamma}-$ заданные выпуклые множества, и, в частности, критерий для вьполнения равенств

$$
\operatorname{conv} \overline{W\left(A_{\gamma},\|\cdot\|_{*}\right)}=\operatorname{conv} \sigma\left(A_{\gamma}\right), \quad \gamma=1, \ldots, n .
$$

Далее

$$
\rho\left(\lambda, \Omega_{\gamma}\right):=\inf _{\mu \in \Omega_{\gamma}}|\lambda-\mu| \quad \text { и } \quad R\left(\lambda, A_{\gamma}\right):=\left(A_{\gamma}-\lambda I\right)^{-1}, \quad \lambda \notin \sigma\left(A_{\gamma}\right) .
$$


Теорема 2. Пусть $A_{1}, \ldots, A_{n}$ - попарно коммутирующие операторы, действующие в банаховом пространстве $B, a \Omega_{1}, \ldots, \Omega_{n}-$ такие замкнутые выпуклые множества, что $\sigma\left(A_{\gamma}\right) \subseteq \Omega_{\gamma}, \gamma=1, \ldots, n$. Тогда для существования такой нормы $\|\cdot\|_{*}$, әквивалентной исходной, что

$$
W\left(A_{\gamma},\|\cdot\|_{*}\right) \subseteq \Omega_{\gamma}, \quad \gamma=1, \ldots, n,
$$

необходимо и достаточно выполнение оченок

$$
\left\|\left(R\left(\lambda, A_{\gamma}\right)\right)^{m}\right\| \leqslant M\left(\rho\left(\lambda, \Omega_{\gamma}\right)\right)^{-m}, \quad \lambda \notin \Omega_{\gamma}, \quad m=1,2, \ldots, \quad \gamma=1, \ldots, n .
$$

ДокАЗАТЕЛьСтво. Необходимость. Пусть существует такая норма $\|\cdot\|_{*}$, что справедливы включения (2). Тогда согласно [7, теорема 6.1] $\left\|R\left(\lambda, A_{\gamma}\right)\right\|_{*} \leqslant\left(\rho\left(\lambda, \Omega_{\gamma}\right)\right)^{-1}$, откуда получаем неравенства (3) в исходной норме $\|\cdot\|$.

Достаточность. Пусть выполнены оценки (3). Соотношениями

$$
\Theta_{\gamma}:=\left\{(\alpha, \beta): \operatorname{Re}(\alpha \omega-\beta) \leqslant 0, \omega \in \Omega_{\gamma}\right\}, \quad \gamma=1, \ldots, n,
$$

введем множества пар комплексных чисел $(\alpha, \beta)$. Каждое множество $\Theta_{\gamma}$ является заостренным конусом в пространстве $\mathbb{C}^{2}$, т.е. из включений $(\alpha, \beta),\left(\alpha^{\prime}, \beta^{\prime}\right) \in \Theta_{\gamma}$ следует справедливость включения $\left(\zeta \alpha+\xi \alpha^{\prime}, \zeta \beta+\xi \beta^{\prime}\right) \in \Theta_{\gamma}$ для произвольных чисел $\zeta, \xi \geqslant 0$. Из определения (4) множеств $\Theta_{\gamma}$ и из предположения о справедливости оценок (3) имеем

$$
\left\|\left(R\left(\lambda, \alpha A_{\gamma}-\beta I\right)\right)^{m}\right\| \leqslant M(\operatorname{Re} \lambda)^{-m}, \quad \operatorname{Re} \lambda>0, \quad m=1,2, \ldots,
$$

при всех $(\alpha, \beta) \in \Omega_{\gamma}$. Отсюда и из [8, теорема 12.3.1] получаем, что $\left\|\exp \left(\alpha A_{\gamma}-\beta I\right)\right\| \leqslant M$, $(\alpha, \beta) \in \Theta_{\gamma}$. Эти неравенства, включение $(0,0) \in \Theta_{\gamma}$ и требование коммутируемости операторов $A_{1}, \ldots, A_{n}$ показьвают, что новая норма $\|\cdot\|_{*}$, заданная равенством

$$
\|x\|_{*}:=\sup _{\left(\alpha_{\gamma}, \beta_{\gamma}\right) \in \Theta_{\gamma}, \gamma=1, \ldots, n}\left\|\left(\exp \left(\sum_{\gamma=1}^{n} \alpha_{\gamma} A_{\gamma}-\beta_{\gamma} I\right)\right) x\right\|,
$$

эквивалентна исходной и $\|x\| \leqslant\|x\|_{*} \leqslant M^{n}\|x\|, x \in B$. Воспользовавшись тем, что каждое из множеств $\Omega_{\gamma}$ является конусом, коммутируемостью операторов $A_{\gamma}$ и определением нормы $\|\cdot\|_{*}$, имеем $\left\|\exp \left(\alpha A_{\gamma}-\beta I\right) t\right\|_{*} \leqslant 1, t \geqslant 0$, для всех пар $(\alpha, \beta) \in \Theta_{\gamma}$. Поэтому согласно [2, теорема 2.1] числовой образ $W\left(\alpha A_{\gamma}-\beta I,\|\cdot\|_{*}\right)$ лежит в полуплоскости $\{\omega: \operatorname{Re} \omega \leqslant 0\}$, как только $(\alpha, \beta) \in \Theta_{\gamma}$, а значит, числовой образ $W\left(A_{\gamma},\|\cdot\|_{*}\right)$ лежит в пересечении полуплоскостей $\{\omega: \operatorname{Re}(\alpha \omega-\beta) \leqslant 0\}$, для которых $(\alpha, \beta) \in \Theta_{\gamma}$. Отсюда из определения (4) множеств $\Theta_{\gamma}$ и из теоремы Минковского [4, c. 13] получаем включения (2).

ЗАмЕЧАнИЕ 2. Если исходное пространство является гильбертовым, а для коммутирующих операторов $A_{1}, \ldots, A_{n}$ справедливы оценки (3), то они не гарантируют существования гильбертовой нормы $\|\cdot\|_{*}$, в которой вьполнены включения (2). Покажем это в случае $n=1$ и множества $\Omega=\Psi_{1}(0)=\{\lambda:|\lambda| \leqslant 1\}$. В работе [9] установлено существование такого оператора $A$, действующего в гильбертовом пространстве и не подобного сжатию, что $\left\|A^{k}\right\| \leqslant c, k=1,2, \ldots$. Значит, для $A$ справедливы оценки (3) с $\Omega=\Psi_{1}(0)$. Предположим теперь существование такой гильбертовой нормы $\|\cdot\|_{*}$, эквивалентной исходной, что в ней выполнено включение $(2)$ с $\Omega=\Psi_{1}(0)$. Поэтому числовой радиус оператора $A$ не превосходит единицы. Отсюда согласно [10, следствие II.8.2] заключаем, что оператор $A$ подобен сжатию в гильбертовой норме $\|\cdot\|_{*}$, тем самым, $A$ подобен сжатию и в исходной норме. Это заключение противоречит выбору $A$. 
ЗАмЕчАнИЕ 3. В случае банахова пространства теорема 1 выводится из теоремы 2 . Для этого достаточно воспользоваться представлением

$$
\left(R\left(\lambda, A_{\gamma}\right)\right)^{m}=-\frac{1}{2 \pi i} \int_{\Gamma_{\gamma}}(\mu-\lambda)^{-m} R\left(\mu, A_{\gamma}\right) d \mu
$$

где каждый из гладких замкнутых контуров $\Gamma_{\gamma}$ выбирается так, что его внутренняя часть содержит спектр оператора $A_{\gamma}$, а сам контур $\Gamma_{\gamma}$ лежит в $\varepsilon$-окрестности спектра оператора $A_{\gamma}, \gamma=1, \ldots, n$.

В связи с теоремой 2 и замечанием 2 возникают две задачи.

ЗАдАчА 1. При каких дополнительных условиях справедлива теорема 2 для бесконечного числа коммутирующих операторов $A_{\gamma}$, действующих в банаховом пространстве?

ЗАДАчА 2. Найти необходимые и достаточные условия, которым должен удовлетворять оператор $A$, действующий в гильбертовом пространстве, чтобы существовала такая гильбертова норма $\|\cdot\|_{*}$, эквивалентная исходной, для которой $W\left(A,\|\cdot\|_{*}\right) \subseteq \Omega$, где $\Omega$ - заданное замкнутое вьпуклое множество, содержашее спектр оператора $A$. Аналогичная задача возникает и для семейства коммутирующих операторов $A_{\gamma}$, действующих в гильбертовом пространстве.

Обе эти задачи интересно решить и в частной их постановке, а именно, когда множества $\Omega_{\gamma}=\operatorname{conv} \sigma\left(A_{\gamma}\right)$.

\section{СПИСОК ЦИТИРОВАННОЙ ЛИТЕРАТУРЫ}

1. Lumer G. // Trans. Amer. Math. Soc. 1961. V. 100. P. 29-43. 2. Lumer G., Phillips R. S. // Pacific J. Math. 1961. V. 11. P. 679-698. 3. Треногин В. А. Функциональный анализ. М.: Наука, 1980. 4. Тихомиров В. М. Выпуклый анализ // Итоги науки и техн. Соврем. пробл. матем. Фундамент. направления. Т. 14. М.: ВИНИТИ, 1987. С. 5-101. 5. Hildebrandt S. // Math. Ann. 1966. V. 163. Р. 230-247. 6. Далецкий Ю. Л., Крейн М. Г. Устойчивость решений дифференциальных уравнений в банаховом пространстве. М.: Наука, 1970. 7. Маркус А. С. // Матем. сб. 1966. Т. 70. № 4. С. 526-561. 8. Хилле Э., Филлипс Р. Функциональный анализ и полугруппы. М.: ИЛ, 1962. 9. Foguel S. R. // Proc. Amer. Math. Soc. 1964. V. 15. P. 788-790. 10. Сёкефальви-Надь Б., Фолш Ч. Гармонический анализ в гилббертовом пространстве. М.: Мир, 1970. 